\title{
Der Weg der Schweizer Psychiatrie ins zwanzigste Jahrhundert
}

Neue Tendenzen und neue Aufgaben der Geschichtsschreibung

anhand der Arbeiten aus den Jahren 1970-1980

\section{Von Hans H. Walser}

Geschichtsschreibung ist, so objektiv sie sich auch geben mag, vom Zeitgeist und von der eingestandenen oder gar nicht selbst wahrgenommenen Ideologie des Historikers abhängig. Entgegen dem Dichterwort steht die Vergangenheit also keineswegs «ewig still», sondern sie erhält ihren Charakter, und oft auch ihren Inhalt, von der Person und der Zeit des Geschichtsschreibers. Bei der Darstellung der Psychiatrie des letzten Jahrhunderts und besonders bei den Entwicklungen und Stillständen in unserem Jahrhundert hat man reichlich Anlaß, dies zu bedenken. Die Prognosen eines H. Bersot ${ }^{1}$ zu Ende $^{2}$ des Zweiten Weltkrieges sind noch recht gemächlich, während sich aus der umfassenden Darstellung von Henri Ellenberger ${ }^{2}$ schon eine kommende Unruhe erahnen läßt. Nach der Konsolidierung der praktischen Psychiatrie um die Jahrhundertwende und nach den eher ruhigen Jahrzehnten, die in Zürich, gerade vor dem Ersten Weltkrieg, nur durch den beunruhigenden, aber auch befruchtenden Kontakt mit der Psychoanalyse unterbrochen war, folgte die durch den Zweiten Weltkrieg und die Nachkriegszeit erzwungene Zurückhaltung. In den letzten zehn oder fünfzehn Jahren ist nun vieles in Bewegung geraten, so daß man sich fragen kann, ob sich nun eine neue «Irrenreform» anbahnt. Dies wird auch den Historiker veranlassen, seine Arbeit neu zu überdenken, ohne daß er deswegen sein Ziel, das Sammeln und vor allem auch die geistige Durchdringung der Fakten der Vergangenheit, vernachlässigen wird.

Vor etwa einem Jahrzehnt haben wir die Hauptlinien der Entwicklung der Schweizer Psychiatrie im letzten Jahrhundert beschrieben ${ }^{3}$. Dazu sind nun einerseits neue Untersuchungen gekommen; anderseits ist auf neue Tendenzen in der Beurteilung der historischen Fakten hinzuweisen.

Der besseren Übersichtlichkeit wegen treffen wir im folgenden die Unterscheidung in «stationäre» und in «ambulante» Psychiatrie - bei voller Kenntnis der Problematik einer solchen Unterscheidung. Zur «stationären 
Psychiatrie» wären die psychiatrische Anstalt, ihr Bau, ihr Betrieb, ihre Möglichkeiten und auch die Hoffnungen zu zählen, die man in sie gesetzt hat, einschließlich natürlich ihrer Forschungsmöglichkeiten und einschließlich der Therapie (aber ausschließlich der rein fürsorgerischen und psychotherapeutischen Bemühungen). Es ist der Teil, den man auch als klinische Psychiatrie, Anstaltspsychiatrie, «Psychiatrie intra muros» und ähnlich bezeichnet hat. Wenn man uns einen Terminus aus der Komputertechnik gestattet, wäre es sozusagen die "psychiatrische Hardware», die dem psychisch Kranken und seinem Arzt angeboten werden kann. Dem wäre (mangels einer geeigneteren Bezeichnung) die «ambulante Psychiatrie» gegenüberzustellen, bei der der Patient in seinen gewohnten Lebensumständen bleibt. Diese Psychiatrie wird meist vom freipraktizierenden Spezialisten oder von den Polikliniken ausgeübt, stellt somit die Therapie in den Vordergrund, umfaßt aber auch alle prophylaktischen und fürsorgerischen Angebote. Eine Behandlung des Kranken gegen seinen Willen oder eine aktive Sicherung vor aggressiven Akten sich selbst oder anderen Personen gegenüber ist bei dieser «Psychiatrie extra muros» nur in einem geringen Maße möglich. Diese Form der Psychiatrie wäre sozusagen die «psychiatrische Software», die sich sowohl der Anstalten, wie auch anderer Strukturen bedienen kann, und so vielleicht auch als «strukturelle psychiatrische Institution» bezeichnet werden kann.

Bei der großen Reform der Psychiatrie um 1800, die zumeist mit dem Namen von Philippe Pinel verknüpft wird, steht nun die «stationäre Psychiatrie» völlig im Vordergrund. Psychiatrie findet in der Klinik statt, zumeist in der staatlichen Anstalt, aber auch in der Privatklinik. Und sogar die praktischen Ärzte, die sich mit Geisteskranken abgaben, nahmen diese oft in ihr Haus auf, so daß sich so etwas wie kleine Privatkliniken konstituierten. Man sollte dieses Faktum nicht mit der absoluten Selbstverständlichkeit zur Kenntnis nehmen, wie es meist geschieht. Eine ausgedehntere, ambulante, fürsorgerische, gesellschaftlich institutionalisierte Psychiatrie wäre auch damals immerhin denkbar gewesen, z. B. von religiösen Gemeinschaften aus. Wahrscheinlich gab es derartiges auch, ohne daß dies aber (bisher?) als wesentliches Element ins historische Bewußtsein gedrungen wäre. Von großem Interesse wäre es, die Ursachen dieses Überwiegens der Anstalt bei der Irrenreform zu untersuchen; zu vermuten ist, daß es sich um ein Erbe des französischen Zentralismus und seines Gesundheitswesens handelt. Der Zentralismus der französischen Könige wurde durch die Revolution ja nicht etwa zerstört, sondern zementiert, und mit ihm 
natürlich auch das Gesundheitswesen. Neben der üblichen «schweizerischen Verspätung», der «mora Helvetica» sämtlicher Kulturbegebenheiten, hat vielleicht auch dies zu einer Verzögerung der Irrenreform in unserem Lande beigetragen: daß nämlich die zentralistische Reformbewegung eines Großstaates zu den klein- und kleinsträumigen Verhältnissen unseres Landes nicht recht passen wollte.

Jedenfalls steht auch bei J.M. Hungerbühler, der 1846 eine Übersicht über das schweizerische Irrenwesen gab und eifrig für deren Reform plädierte, die Anstalt ganz im Vordergrund ${ }^{4}$. Sie bedeutete ja auch einen gewaltigen, humanitären und medizinischen Fortschritt. In jener Zeit entwickelte sich ein bestimmter Typus der psychiatrischen Anstalt größtenteils wohl aus dem Ausland übernommen, zum kleineren Teile bei uns modifiziert -, der jedenfalls bis heute Bestand hat. Zu diesem Anstaltstypus gehören zunächst die Trennung der Irren von den körperlich Kranken, den Invaliden, Versorgungsbedürftigen und Kriminellen; die Einrichtung eigener Irrenanstalten außerhalb der Städte, sei es in Neubauten oder in aufgehobenen Klöstern; ein voller Primat der ärztlichen Leitung gegenüber dem bisher herrschenden Verwaltungsbeamten; eine möglichste Integrierung des Irrenwesens in Medizin und Medizinalwesen. Bei der Einrichtung der Anstalten war die Geschlechtertrennung ein absolutes Prinzip; Abteilungen mit Spazierhöfen sorgten für eine Trennung der Geisteskranken nach ihrem Verhalten (weniger nach der ohnehin oft nur rudimentären Diagnose). Der Betrieb der Anstalten war in seiner Qualität unterschiedlich, teilweise aber erstaunlich modern, namentlich dort, wo das Non-Restraint-System voll durchgeführt und wo auf möglichste Öffnung der Anstalt geachtet wurde $^{5}$. Vorläufer dieser Reform findet man in Genf 1838 und Basel 1842; ihre volle Ausprägung fand sie z. B. in Bern 1855 und in Zürich 1870. Diese gewaltige Reformarbeit wäre ohne die Hilfe hervorragender deutscher Psychiater nicht möglich gewesen ${ }^{6}$.

$\mathrm{Zu}$ jedem der Merkmale des Anstaltstypus ließen sich nun eingehende kritische Bemerkungen machen. Dabei muß man sich aber sehr hüten, frühere Zustände an heutigen Maßstäben zu messen, denn nach $150 \mathrm{Jahren}$ ist man doch hoffentlich gescheiter als vorher. Damit darf man sich aber nicht einfach beruhigen, denn es gibt wenigstens ein hervorragendes Zeugnis, daß auch damals eine andere Form der Irrenpflege bereits denkbar war und auch hätte durchgeführt werden können. Es sind dies die Griesingerschen Vorschläge von 1868, die bereits eine weitgehend «offene Psychiatrie» hätten initiieren können ${ }^{6}$. Das gibt uns die Berechtigung, nach den damals 
bereits bestehenden gedanklichen Möglichkeiten zu fragen und das Verwirklichte daran zu messen. Das heißt natürlich nicht, daß nun die ganze Geschichtsschreibung umgekrempelt werden müsse. Wohl aber wird man gewisse Akzente anders setzen und auf Fragen stoßen, an die man vorher gar nicht gedacht hat. In der folgenden Übersicht sollen einige solche Hinweise gegeben werden.

Erfreulich ist, daß seit 1970 eine ganze Reihe von Arbeiten zur Psychiatrie des 19. und des 20. Jahrhunderts erschienen sind. Was die psychiatrischen Anstalten betrifft, so liegt seit 1972 eine reichhaltige, gut illustrierte Zentenarschrift über die Klinik Königsfelden vor ${ }^{7}$. Im folgenden Jahre wurde ein ähnlich konzipiertes Buch über die Klinik Cery bei Lausanne vorgelegt $^{8,9}$. Als Zürcher Dissertation ist die Geschichte der luzernischen Anstalt St. Urban erschienen ${ }^{10}$. Eine Geschichte der Anstalt St. Pirminsberg befindet sich in Vorbereitung. - Das reiche Material dieser Arbeiten ist chronologisch geordnet und beleuchtet die Vergangenheit hauptsächlich aus der Sicht der Ärzte und der verwalterischen Leitung. Was man aber besonders gerne wüßte, ist, wie der Klinikaufenthalt vom einzelnen Patienten erlebt wurde. Es hat sich ja in den Anstalten eine eigentliche «Subkultur» entwickelt. Ohne daß man nun mit allem einverstanden sein müßte, sollte man vor der Niederschrift einer Klinikgeschichte vielleicht doch Arbeiten wie diejenige von E.Goffman ${ }^{11}$ und F. Fischer ${ }^{12}$ als Denkanstöße lesen. Natürlich ist es schwierig, das Anstaltserlebnis eines Patienten oder ein Stück der «Psychiatrischen Subkultur» zu erfassen. So wird es wohl nicht allzu viele schriftliche Berichte von Patienten geben, und die Krankengeschichten sind oft, wie schon $\mathrm{H}$. Ellenberger bemerkte, belanglos. Zeugnisse der bildenden Kunst sind nicht nur häufiger, sie sind auch sehr in Mode gekommen, wobei ihre Bearbeiter aber in einer anderen Richtung interessiert sind. Suchen sollte man aber nach solchen mündlichen und schriftlichen Äußerungen von Patienten, und man sollte wohl auch die Produkte der bildenden Kunst auf das Befinden des Kranken und auf sein Anstaltserlebnis hin befragen. Wie sehr das Leben in der Subkultur der Anstalt von der Realität entfernt war, zeigt z. B. das Prinzip der Geschlechtertrennung, das sogar für den fortschrittlichen Griesinger noch selbstverständlich war. Das kam auf eine Negierung und auf ein «Verbot» der Sexualität heraus. Ganz ähnlich wurde mit dem weiteren menschlichen Grundtrieb, der Aggressivität, umgegangen ${ }^{13}$.

Biographische Darstellungen erschienen über Ludwig Wille (1834-1912), den deutschen Psychiater, dem die Schweizer Psychiatrie zu besonderem 
Dank verpflichtet ist ${ }^{14}$. Der Vergleich mit Friedrich Brenner (1809-1874) ist auch darum interessant, weil er zeigt, was die Schweiz aus eigenen Kräften hat leisten - oder eben nicht leisten können ${ }^{15}$. Dasselbe zeigt die Darstellung von J. J. Jörger (1860-1933), aber nun doch eher im umgekehrten Sinn: wie es nämlich einem Psychiater in einer Landanstalt möglich sein konnte, wesentliche wissenschaftliche Arbeit zu leisten ${ }^{16}$. Sehr dankbar muß man schließlich sein, daß M. Cramer die bisher etwas rätselhafte Rolle des Genfers Abraham Joly (1748-1812) bei der frühen Irrenreform klären konnte ${ }^{17}$. Auch August Zinn ist kurz gewürdigt worden ${ }^{18}$. Solchen Arbeiten haftet natürlich die Schwierigkeit jeglicher Biographik an. Gerne wüßte man mehr über einen solchen Mann, über seine Persönlichkeit, seine Emotionalität, sein Motiv zur Wahl gerade dieses Berufes, über seine soziale Kontaktfähigkeit, seine innere Einstellung zu den Patienten. Überhaupt: wie sah das Leben eines dieser Psychiater denn aus? Wie wurde es geformt durch die neuen äußeren Bedingungen, so etwa durch die Möglichkeiten und Zwänge des Anstalttypus? Um ein extremes Beispiel zu erwähnen: Gustav Bally erzählte von einem seiner Chefs, daß er jeweils mit Begeisterung den Zuchtstier der anstaltseigenen Landwirtschaft gemustert habe und dabei geradezu aufgeblüht sei, während er mit seinen Patienten nicht viel anfangen konnte (er wurde denn auch, von ganz anderer Seite, ähnlich literarisch dargestellt!). Hat sich da vielleicht der ärztliche Primat, der in vielem doch wohltätig war, an seinen Urhebern gerächt, indem er einzelnen Ärzten nicht nur die Kompetenzen, sondern auch das Wohlgefallen an administrativer Arbeit gab? Kurz: man würde «Psychogramme» der Psychiater, und zwar solche, die die Verflechtung mit ihrer Anstalt mit aufzeigen, wünschen. Von einem Doktoranden das zu verlangen, wäre reine Überforderung. Schließlich ist man froh, wenn man einmal die genauen Lebensumstände, das Werkverzeichnis und den Hinweis auf weitere Quellen hat. So wurde jedenfalls darauf geachtet, daß direkte Zeugnisse von Kollegen, Freunden und Familienmitgliedern berücksichtigt werden mußten. Nun kommen gewisse Autobiographien einem Psychogramm manchmal recht nahe. August Forel ist hier eine große Einzelerscheinung geblieben. In die Nähe seines Werkes könnte bisher wohl nur die Selbstbiographie von Max Müller (früher Professor für Psychiatrie in Bern) gerückt werden. Jedoch würden auch die Aufzeichnungen von Karl Gehry eine Edition in Auswahl lohnen (s.u.). Daß schließlich eine Biographie über Eugen Bleuler ein großes Desiderat wäre, ist wohl selbstverständlich.

Regionale Darstellungen sind bei uns am ehesten gemäß den Sprachgebie- 
ten zu erwarten. So liegen zwei kleinere Arbeiten über die Psychiatrie in der Romandie vor ${ }^{19,20}$.

Das Mit- und Gegeneinander wissenschaftlicher und anstaltstechnischer Probleme hat E. H. Ackerknecht am Beispiel des «Burghölzli-Skandals» und seines Umfeldes von 1869-1879 dargestellt ${ }^{21}$. Diese turbulente Periode in Zürich ist so bedeutend, weil aus ihr schließlich eine Schweizer Psychiatrie eigener Prägung hervorging.

Allmählich wurde auch in der Anstaltspsychiatrie der Trend zur ambulanten Psychiatrie stärker. Er zeigt sich z. B. in der Schaffung von Außenstationen, und insbesondere in den Hilfsvereinen für entlassene Geisteskranke, die oft schon in den siebziger Jahren bestanden. Hier liegt - außer bei Griesingers Nachwirkung - ein weiterer Ansatz zur Sozialpsychiatrie. Der Zürcher Hilfsverein für Geisteskranke ist dafür ein wichtiger Beleg ${ }^{22}$.

Die starken sozialethischen Impulse bei August Forel haben gewiß tendenziell das Aufkommen der Sozialpsychiatrie («avant la lettre») begünstigt. So ist es wohl kein Zufall, daß gerade sein Schüler Adolf Meyer in den USA die Bewegung der «Mental Hygiene» mittrug. Sodann hat die Sozialethik gewiß zur Hinwendung Forels zur politischen Linken beigetragen (die heutige Linke läßt es sich zwar eher angelegen sein, diesen Mann in beinahe schon verleumderischer Weise mißzuverstehen!). Dieser politisch-soziale Fragenkomplex wäre jedenfalls einer kompetenten, sachlichen Untersuchung wert. - Auch Forels Kampf gegen den Alkoholismus dürfte, auf dem Hintergrund der heutigen Drogenszene, zu einer Neubewertung Anlaß geben.

Besonders folgenreich war schließlich die Aufnahme des Hypnotismus in das wissenschaftlich-psychiatrische Forschungsgebiet, das August Forel (1848-1931) in den achtziger Jahren vollzog. Er strebte ein vertieftes psychologisches Verstehen des Patienten und vermehrte Berücksichtigung der Psychotherapie an; ob er aber schon ahnte, welche Kräfte damit in Bewegung gesetzt wurden, ist fraglich. Jedenfalls wurden neurotische Krankheiten erst durch die Hypnose und die Suggestionstherapie einer spezialisierten ärztlichen Therapie zugänglich, und dann ließ ja auch die Psychoanalyse nicht mehr lange auf sich warten. So wurde von der Jahrhundertwende an die psychiatrische Privatpraxis erst möglich, und im Verlauf von zwei oder drei Jahrzehnten wurde sie zu einer Institution der «Psychiatrie extra muros». Ohne die Vorbereitung und die innere Sensibilisierung durch Forels Hypnosestudien wäre das frühe, wache und verständnisvolle Interesse von Eugen Bleuler (1857-1939) und C. G. Jung (1875-1961) für die Psychoanalyse kaum denkbar. 
Überhaupt hat die Psychoanalyse in der Schweizer Psychiatrie eine große Rolle gespielt - und umgekehrt hatte die Zürcher Psychiatrie für die Vorgeschichte und die Frühgeschichte der Psychoanalyse eine sehr große Bedeutung. Sigmund Freud ging ja von psychophysischen Gedankengängen aus, wie sie damals viele Forscher beschäftigten. Treibende Kräfte waren einerseits ein wiedererwachtes psychologisches Interesse (wie es ja in der Hypnosebewegung zum Ausdruck kam), anderseits die großen Erfolge der Anatomie und Histologie des Gehirnes, die unter anderem zur Neuronentheorie führten. Man könnte geradezu versucht sein, von einer «psychoanatomischen» Forschungsrichtung zu sprechen. August Forel hat sich daran insbesondere mit seinen Arbeiten über das Gedächtnis (1885) sowie über Gehirn und Seele (1894) intensiv beteiligt ${ }^{23,24}$.

August Forel hat zwar noch die frühe psychokathartische Methode von Josef Breuer und Sigmund Freud (1895) akzeptiert, mit der weiteren Entwicklung der Psychoanalyse konnte er sich aber in keiner Weise befreunden. Das blieb seinem Schüler Eugen Bleuler vorbehalten, der als Professor und Klinikdirektor in Zürich, zusammen mit seinem Oberarzt C. G. Jung, mit Sigmund Freud in eine so enge Beziehung trat, daß man beinahe von einer mehrjährigen Arbeitsgemeinschaft (1906-1913) sprechen kann. So bildet denn der Briefwechsel zwischen Freud und Jung auch für die Schweizer Psychiatrie eine wichtige Quelle ${ }^{25}$. Dasselbe gilt natürlich auch für die Erinnerungen von C. G. Jung ${ }^{26}$ sowie in einem gewissen Maße auch für diejenigen von Herman Nunberg ${ }^{27}$ und für die Biographie über Jungs Kollegen und Rivalen Karl Abraham ${ }^{28}$, und insbesondere auch für die schon früher erschienene, sympathische Freud-Darstellung von Ludwig Binswanger ${ }^{29}$. Wie sehr die damals heranwachsenden Schweizer Psychiater von der Psychoanalyse beeinflußt waren, hat z. B. H. Ellenberger in seiner Darstellung von Hermann Rorschach gezeigt ${ }^{30,31}$. Daß dies eine allgemeine Erscheinung war, belegen Namen wie Philipp Sarasin ${ }^{32}$, Arthur Kielholz ${ }^{33}$, Karl Gehry ${ }^{34}$ und insbesondere Max Müller ${ }^{35}$, Hans Christoffel ${ }^{36}$ und Rudolf Brun ${ }^{37}$. Diese Beeinflussung betraf keineswegs nur die ambulante Psychiatrie, sondern besonders auch die stationäre, da die meisten der Genannten an leitender Stelle in Kliniken tätig waren. Auch zur «tragischen Geschichte» von Psychiatrie und Psychoanalyse hat die Schweiz beigetragen, wie Arbeiten über Johann Jakob Honegger ${ }^{38,39}$ und über Otto Groß ${ }^{40}$ zeigen. Daß die frühe Rezeption der Psychoanalyse eine der Eigenarten der Schweizer Psychiatrie ist, zeigt bereits ein kurzer Überblick ${ }^{41}$, der erfreulicherweise weiteres Interesse erweckte ${ }^{42}$ und zu neuen Quellen führte. 
Weitere Desiderate wären etwa: Arbeiten über diejenigen Kliniken, die einer historischen Darstellung noch nicht teilhaftig wurden (einiges ist vorgesehen), sowie die Geschichte der psychiatrischen Polikliniken (auch hier gibt es Anfänge, so für Basel ${ }^{43}$ ). Auch vermehrte biographische und autobiographische Darstellungen hätte man gerne. Natürlich gibt es auch hier einige neuere Beiträge, so über Jakob Klaesi ${ }^{44}$ und Gustav Wolff ${ }^{45}$. Dann enthält das Sammelwerk von Pongratz autobiographische Artikel von Hans Binder, Jakob Klaesi, Jakob Wyrsch und Roland Kuhn ${ }^{46}$. Erfreulich ist, daß Oscar Forel sich entschließen konnte, seine autobiographischen Aufzeichnungen herauszugeben ${ }^{47}$. Sehr gerne sähe man die Selbstdarstellung von Karl Gehry wenigstens auszugsweise und diejenige von Max Müller möglichst vollständig gedruckt. Gerade diese letztere Autobiographie ist wohl eine der wichtigsten Quellen für die Schweizer Psychiatrie der Zwischen- und der zweiten Nachkriegszeit.

Bis über den Zweiten Weltkrieg hinaus ging die Anstaltspsychiatrie trotz mancher Verbesserungen und Einzelleistungen - ihren eher gemächlichen Gang. Die ambulante Psychiatrie und die eigentliche Psychotherapie hatten sich weitgehend in die private Praxis zurückgezogen. Verbindungen fehlten zwar nicht ganz, blieben aber ohne große Wirkung. Einen Neubeginn verdankt man Manfred Bleuler, der 1951, nach einem Arbeitsaufenthalt in den USA, Gustav Bally und Medard Boß mit der psychotherapeutischen Ausbildung seiner Ärzte in der psychiatrischen Universitätsklinik Zürich betraute. Hier wurde das «Institut für ärztliche Psychotherapie» gegründet, welches unter aktiver Beteiligung von jüngeren Psychiatern (Fritz Meerwein, Gaetano Benedetti, Christian Müller, Hans Kind, Balthasar Staehelin, Gion Condrau, Hans-Konrad Knoepfel u. a.) die Psychiatergeneration, die in den fünfziger und sechziger Jahren ihre Ausbildung in Zürich genoß, wesentlich beeinflußte. Weitere Kliniken folgten dem Beispiel, so namentlich Basel, Bern und Lausanne, während in Genf der gleichfalls aus den USA zurückgekehrte Psychoanalytiker Raymond de Saussure psychoanalytische Vorlesungen hielt (worin er erfreulicherweise der Geschichte der Psychiatrie einen besonderen Platz zuwies!). Daß hier wirklich ein neuer Anlauf genommen wurde, zeigt sich etwa im Vergleich mit der obenerwähnten, genauen Bestandesaufnahme der Zeit bis 1950 von Henri Ellenberger, auf die nochmals verwiesen sei.

Gewiß bedeutete dieser Neubeginn eine verstärkte Durchdringung von «stationärer» und «ambulanter» Psychiatrie, die institutionell auch in einer Betonung der (staatlichen) Polikliniken als Brücke zwischen beiden zum 
Ausdruck kam (unter anderem durch Dotierung der Polikliniken mit Bettenstationen). Seit kurzem ist auch eine gewisse psychotherapeutische Ausbildung des angehenden Fachpsychiaters obligatorisch geworden. Diese Zeit liegt so kurz zurück, daß sie im vergangenen Jahrzehnt, über das ja hier berichtet wird, noch kaum einen historischen Niederschlag gefunden hat. Freilich wäre es wünschenswert - sogar sehr!-, daß auch auf unserem Gebiet die Zeitgeschichte vermehrt berücksichtigt würde.

Eine weitere, sehr wesentliche Wandlung erfuhr die Institution klinische Psychiatrie durch die Einführung neuer Psychopharmaka seit 1951. Bezeichnend ist, daß dies die Position der ambulanten Psychiatrie nicht geschwächt hat; es ergab sich vielmehr ein fast selbstverständliches Zusammenspiel beider Gebiete.

Versucht man einen Ausblick, so kann man nicht umhin, auf die heute herrschende Welle psychotherapeutischer Bestrebungen und Erscheinungen hinzuweisen. Die stark veränderte und oft politisierte Neuauflage der sogenannten «Antipsychiatrie» hat unser Land natürlich auch erreicht. Das neue Interesse für die Psychotherapie hat ein sehr weites und ein weit über Psychiatrie und ärztliche Psychotherapie hinausreichendes Spektrum. Es reicht von sehr erwünschten medizinisch-wissenschaftlichen Tendenzen (wie Psychosomatik, Psychotherapie der Psychosen, Psychotherapie in der Allgemeinpraxis und vieles mehr) über ein weites Gebiet populären Interesses bis hin zur Kurpfuscherei («Psycho-Pfuscherei») und zu menschlich abstoßenden Erscheinungen, auch zu solchen durchaus institutioneller Art (es braucht wohl nicht betont zu werden, daß diese «Institutionen» mit den hier behandelten keine Beziehung haben und keinen Vergleich ermöglichen). Es ist aber darauf hinzuweisen, daß dieser populäre und von politischen Kreisen gelegentlich mißbrauchte «Psycho-Boom» auch auf Medizin und Psychiatrie seine Auswirkungen hat - sei es in der Abwehr, sei es in der Beachtung oder gar Übernahme einzelner richtiger Ideen. Aber es war ja schon immer so, daß sich die Medizin im staatlichen, sozialen und politischen Zusammenhang entwickelte, und das gilt für die Psychiatrie als mannigfaches Grenzgebiet zwischen dem Körper und der Seele, zwischen dem Staat und dem einzelnen, zwischen Medizin und Philosophie, zwischen Natur- und Geisteswissenschaften ganz besonders. 


\section{Literatur}

${ }^{1}$ Bersot, H., Destins de la psychiatrie suisse, Berne, s. d. (env. 1945).

${ }^{2}$ Ellenberger, Henri, La psychiatrie suisse, Aurillac 1951/53.

${ }^{3}$ Walser, Hans H., Schweizer Psychiatrie im 19. Jahrhundert, Gesnerus 29 (1972), 183-195 (dort auch die frühere Literatur bis etwa 1970).

${ }^{4}$ Hungerbühler, J. M., Über das öffentliche Irrenwesen in der Schweiz, St.Gallen und Bern 1846.

${ }^{5}$ Geduldig-Jacki, Cordula, Die Behandlung von Geisteskranken ohne physischen Zwang, Diss. med. Zürich 1975.

${ }^{6}$ Walser, Hans H., Die «deutsche Periode» (etwa 1850-1880) in der Geschichte der Schweizer Psychiatrie und die moderne Sozialpsychiatrie. Gesnerus 28 (1971), 47-55.

${ }^{7}$ Bressler, Hans G., Königsfelden 1872-1972, Aarau o. J. (1972).

${ }^{8}$ Secrétan, Claude, L'hôpital de Cery 1873-1973, Lausanne 1973.

${ }^{9}$ Müller, Christian, Le Champ-de-l'Air, premier hôpital psychiatrique du Canton de Vaud. Gesnerus 37 (1980), 28-33.

${ }^{10}$ Baumann, Urs, Kantonale Psychiatrische Klinik St.Urban 1873-1973, Diss. med. Zürich 1973 (und St. Urban, Kt. Luzern 1973).

${ }^{11}$ Goffman, Erving, Asyle, Frankfurt a. M. 1972 (edition suhrkamp 678).

${ }^{12}$ Fischer, Frank, Irrenhäuser, München 1969.

${ }^{13}$ Walser Hans H., Geschichtliche Anmerkungen zum Problem der Aggressivität in der klinischen Psychiatrie. Schweizer Archiv für Neurologie, Neurochirurgie und Psychiatrie 128 (1981), Heft 2.

14 Meyer, Christian, Ludwig Wille (1834-1912), Diss. med. Zürich 1973.

${ }^{15}$ Schüler, Georges, Der Basler Irrenarzt Friedrich Brenner 1809-1874, Aarau 1974 (und Diss. med. Zürich 1974).

16 Mornaghini-Zweidler, Liliana, Der Psychiater Johann Joseph Jörger (1860-1933), Diss. med. Zürich 1975.

${ }^{17}$ Cramer, Marc, Un précurseur peu connu de Pinel: le médecin genevois Abraham Joly (1748-1812). Médecine et Hygiène, No.1118, 9 octobre 1974, S.1572-1573.

18 Walser Hans H., und Zinn, Wilhelm M., August Zinn (1825-1897), ein Begründer der praktischen Psychiatrie in der Schweiz. Gesnerus 32 (1975), 271-282.

19 de Morsier, Georges, Histoire de la psychiatrie et de la neurologie à Genève. Gesnerus 34 (1977), 186-197.

${ }^{20}$ Walser, Hans H., Zur Psychiatrie in der Westschweiz im 19. Jahrhundert. Gesnerus 32 (1975), 182-191. - Nekrolog R. de Saussure. Gesnerus 30 (1973), 177-180.

${ }^{21}$ Ackerknecht, Erwin H., Gudden, Huguenin, Hitzig. Hirnpsychiatrie im Burghölzli 1868-1879. Gesnerus 35 (1978), 66-78.

${ }^{22}$ Ausfeld, Rudolf, Anfänge der sozialpsychiatrischen Bestrebungen im Kanton Zürich, Diss. med. Zürich 1977.

${ }^{23}$ Walser, Hans H., Über Theorien des Gedächtnisses in den letzten Dezennien des 19. Jahrhunderts und ihre Bedeutung für die Entstehung der Psychoanalyse. Proceedings of the XXIII Congress of History of Medicine, London 1972, 1227-1232.

${ }^{24}$ Bialas, Angelika, Ansätze zu einer dynamischen Psychologie bei August Forel. Ein Beitrag zur Vorgeschichte der Psychoanalyse. Diss. med. Zürich 1974 (Arbeit unter der Leitung von Prof. H. Walser). 
${ }^{25}$ McGuire, William, und Sauerländer, Wolfgang (Hrsg.), Sigmund Freud/C.G. Jung. Briefwechsel. Frankfurt a. M. 1974.

${ }^{26}$ Jaffé, Aniela, Erinnerungen, Träume, Gedanken von C. G. Jung, Zürich 1962.

${ }^{27}$ Nunberg, Herman, M. D., Recollections, Ideas, Reflections, New York 1969.

${ }^{28}$ Abraham, Hilda, Karl Abraham. Sein Leben für die Psychoanalyse. München 1976.

${ }^{29}$ Binswanger, Ludwig, Erinnerungen an Sigmund Freud, Bern 1956.

${ }^{30}$ Ellenberger, Henri F., Les mouvements de libération mythique et autres essais sur l'histoire de la psychiatrie, Montréal/Québec 1978 (229-289: La vie et l'œuvre de Hermann Rorschach, 1884-1922).

${ }^{31}$ Rorschach, Hermann, Ausgewählte Aufsätze (Hrsg. K. W.Bash), München o. J. (ca. 1970).

${ }^{2}$ Philipp Sarasin, der spätere langjährige Präsident der Schweizerischen Gesellschaft für Psychoanalyse, war 1916-1921 in der Anstalt Rheinau tätig, von wo er zu Sigmund Freud nach Wien zur Lehranalyse ging (vgl.34).

${ }^{33}$ Einige wenige Angaben über Arthur Kielholz finden sich in 7 und 34.

${ }^{34}$ Walser, Hans H., Hundert Jahre Klinik Rheinau, Aarau 1971. (Karl Gehry war 1905 in die Anstalt Rheinau als Assistenzarzt eingetreten; er verließ die Klinik 1942 als Direktor. Seine in vieler Hinsicht aufschlußreiche, umfängliche Autobiographie befindet sich in Familienbesitz.)

${ }^{35}$ Die Autobiographie von Max Müller befindet sich in Familienbesitz. Sie ist nicht zuletzt so wertvoll, weil Max Müller nicht nur bei Eugen Bleuler Assistenzarzt war, sondern sich bei Emil Oberholzer einer Psychoanalyse unterzog und in der Geschichte der Schweizer Psychoanalyse eine Rolle spielte. Zudem hat er seine ungeschminkte, wenn auch sachliche Meinung über Personen und Ereignisse gesagt. Dies hat wohl dazu beigetragen, daß er seine Autobiographie zu Lebzeiten nicht mehr herausgeben wollte, um so mehr als er sie auch nicht gekürzt oder «gemildert» sehen wollte.

${ }^{36}$ Eine Dissertation über Hans Christoffel befindet sich in Vorbereitung.

${ }^{37}$ Aeschlimann, Jürg, Rudolf Brun (1885-1969). Leben und Werk des Zürcher Neurologen, Psychoanalytikers und Entomologen. Diss. med. Zürich 1980.

${ }^{38}$ Walser, Hans H., Johann Jakob Honegger (1885-1911). Ein Beitrag zur Geschichte der Psychoanalyse. Schweizer Archiv für Neurologie, Neurochirurgie und Psychiatrie 112 (1973), 107-113.

${ }^{39}$ Walser, Hans H., An Early Psychoanalytical Tragedy. J. J. Honegger and the Beginning of Training Analysis. Spring 1974. An Annual of Archetypal Psychology and Jungian Thought. New York City 1974, S. 243-255.

${ }^{40}$ Hurwitz, Emanuel, Otto Gross. Paradies-Sucher zwischen Freud und Jung. Zürich 1979.

${ }^{41}$ Walser, Hans H., Psychoanalyse in der Schweiz. In: Die Psychologie des 20. Jahrhunderts, Band II (Hrsg. Dieter Eicke), Zürich 1976, S. 1192-1218.

${ }^{42}$ Meerwein, Fritz, Reflexionen zur Geschichte der Schweizerischen Gesellschaft für Psychoanalyse in der deutschen Schweiz. Mitteilungsblatt der Schweiz. Gesellschaft für Psychoanalyse No.9, o. O. 1979.

${ }^{43}$ Haenel, Thomas, Zur Geschichte der Psychiatrischen Poliklinik Basel. Schweizer Archiv für Neurologie, Neurochirurgie und Psychiatrie 124 (1979), 335-350.

${ }^{44}$ Haenel, Thomas, Jakob Klaesi - Schlafkur und Antieidodiathese. Gesnerus 36 (1979), 246-265.

${ }^{45}$ Haller, Hans-Rudolf, Gustav Wolff (1865-1941) und sein Beitrag zur Lehre vom Vitalismus, Diss. med. Basel 1968. 
${ }^{46}$ Pongratz, Ludwig J., (Hrsg.), Psychiatrie in Selbstdarstellungen, Bern/Stuttgart/Wien 1977. - Vgl. auch Gesnerus 36 (1979), $319 f$.

${ }^{47}$ Forel, Oscar, La mémoire du chêne, Lausanne 1980. - Vgl. auch Gesnerus 37 (1980), 160-162.

\section{Summary}

Between 1970 and 1980 new contributions have been made to the history of Swiss psychiatry in the 19th and in the early 20 th century. These papers are presented here, together with some new theoretical aspects to the historiography of this period.

Prof. Dr. med. Hans H. Walser

Medizinhistorisches Institut

Rämistraße 71

CH-8006 Zürich 